\title{
Identification of Arabidopsis Mutants Exhibiting an Altered Hypersensitive Response in Gene-for-Gene Disease Resistance
}

\author{
I-ching Yu, Kevin A. Fengler, Steven J. Clough, and Andrew F. Bent \\ Department of Crop Sciences, University of Illinois at Urbana-Champaign, Urbana 61801, U.S.A. \\ Accepted 22 November 1999.
}

\begin{abstract}
A mutational study was carried out to isolate Arabidopsis thaliana plants that exhibit full or partial disruption of the RPS2-mediated hypersensitive response (HR) to Pseudomonas syringae that express avrRpt2. Five classes of mutants were identified including mutations at RPS2, dnd mutations causing a "defense, no death" loss-of-HR phenotype, a lesion-mimic mutant that also exhibited an $\mathrm{HR}^{-}$ phenotype, and a number of intermediate or partial-lossof-HR mutants. Surprisingly, many of these mutants displayed elevated resistance to virulent $P$. syringae and, in some cases, to Peronospora parasitica. Constitutively elevated levels of pathogenesis-related (PR) gene expression and salicylic acid were also observed. In the lesion-mimic mutant, appearance of elevated resistance was temporally correlated with appearance of lesions. For one of the intermediate lines, resistance was shown to be dependent on elevated levels of salicylic acid. A new locus was identified and named IHRI, after the mutant phenotype of "intermediate HR." Genetic analysis of the intermediateHR plant lines was difficult due to uncertainties in distinguishing the partial/intermediate mutant phenotypes from wild type. Despite this difficulty, the intermediate-HR mutants remain of interest because they reveal potential new defense-related loci and because many of these lines exhibit partially elevated disease resistance without dwarfing or other apparent growth defects.
\end{abstract}

Additional keywords: dnd1, dnd2.

The cloning of plant disease resistance genes, pathogen avirulence genes, and other defense-associated genes has presented new opportunities to understand the pathogen recognition and defense signal transduction processes that underlie disease resistance. While study of these known genes and proteins is in progress, the identification of additional compo-

Corresponding author: Andrew F. Bent; Fax: 608-263-2626

E-mail: afb@plantpath.wisc.edu

Current address of I-ching Yu: Department of Biology, University of North Carolina, Chapel Hill 27599, U.S.A.

Current address of Kevin Fengler: DuPont Agricultural Biotechnology, Wilmington, DE 19880, U.S.A.

Current address of Andrew Bent: Department of Plant Pathology, University of Wisconsin, Madison 53706, U.S.A. nents of plant defense pathways remains a high priority. Mutational strategies have been particularly effective for the identification of genes that influence plant defense. For example, mutants of Arabidopsis thaliana have been used by a number of researchers to identify genes involved in plant defense (Dong 1998; Innes 1998).

In earlier screens that investigated the resistance of Arabidopsis to $P$. syringae that express avrRpt2, seven mutant alleles of RPS2 were found but no loci other than RPS2 were identified (Kunkel et al. 1993; Yu et al. 1993). There are many possible explanations for this recurrent identification of rps 2 mutations to the exclusion of other genes in the avrRpt2-RPS2 response pathway. Lethality of functional disruption could prevent identification of other relevant mutations. Genetic redundancy could also limit gene identification. If defense activation pathways are very short, few additional loci may exist to be identified. Genes that function after a branch point in defense induction and control a subset of downstream defense responses could easily escape detection if their contribution to resistance is only quantitative or partial. Lastly, recovery of a relevant class of mutants might be precluded by the particular screening method used. Screening of larger populations might be all that is required to uncover mutations in other loci of the avrRpt2-RPS2 pathway. As an alternative, the present mutational study addressed these issues through a modified screening procedure (Yu et al. 1998) that was sensitive to partial-loss-of-function mutations. This screen monitored the presence and timing of the hypersensitive response (HR).

The HR is a programmed cell death process intimately associated with the defense response mediated by plant resistance genes and pathogen avirulence genes (Bent 1996; Dangl et al. 1996; Goodman and Novacky 1994; Hammond-Kosack and Jones 1996; Richberg et al. 1998). Beneficial roles of the HR include the elimination of host cells that support pathogen growth, the release of antimicrobial compounds, and the release of signals that activate defense responses in neighboring and distant host cells. The HR is not always required for effective gene-for-gene resistance (Bendahmane et al. 1999; Goulden and Baulcombe 1993; Lehnackers and Knogge 1990; Ori et al. 1997; Schiffer et al. 1997; Yu et al. 1998). However, the above-noted benefits of HR cell death are apparently important in many gene-for-gene interactions, and useful information is expected from further study of the relationship between HR cell death and activation of host defenses. 
We recently reported the identification of Arabidopsis DNDl (Yu et al. 1998). Arabidopsis plants homozygous for the recessive dndl-1 mutation exhibit a "defense, no death" phenotype, in which plants exhibit strong gene-for-gene resistance to avirulent $P$. syringae despite the virtual absence of HR cell death. Mutant $d n d l$ plants also exhibit a degree of constitutive resistance to virulent $P$. syringae, Peronospora parasitica, and Tobacco ringspot virus, similar to the defense observed in plants induced for systemic acquired resistance. This constitutive resistance is quantitatively less effective than the inducible gene-for-gene resistance. The dndl mutant, along with some Arabidopsis $l s d, a c d, c p r$, and cim mutants, fits into a larger class of mutated lines that exhibit a dwarfed rosette, elevated levels of salicylic acid (SA), and constitutively heightened resistance to virulent pathogens. These mutants differ from each other by the presence or absence of lesion mimicry, by their HR phenotypes, by leaf shape, glabrousness and other morphological phenotypes, and by the different loci that are responsible for the mutant traits.

The present report describes additional Arabidopsis mutants isolated based on their altered HR in response to $P$. syringae that express avrRpt2. Like $d n d 1$, many of these mutants exhibit elevated resistance to virulent pathogens as well as a reduced HR in response to avirulent $P$. syringae.

\section{RESULTS}

\section{Mutant screen.}

A mutational approach was pursued to identify components of the avrRpt2-RPS2 disease resistance pathway in Arabidopsis. Plants were inoculated with $P$. syringae that express avrRpt2 (Dong et al. 1991; Whalen et al. 1991). Artificially high titers of pathogen were used to induce confluent, macroscopically observable HR cell collapse (Klement et al. 1964). Screens for loss of HR had previously been used to identify mutant alleles of RPS2 (Yu et al. 1993), but for the present work we designed a new method that allowed high throughput screening with detection of intermediate or delayed-HR phenotypes (see also Kunkel et al. 1993; Yu et al. 1998). A delayed HR can be difficult to detect if plants are inoculated with virulent $P$. syringae that express an $a v r$ gene because at high inoculum titers the virulence of these strains leads to killing and necrosis of susceptible plant cells roughly 36 to 48 $\mathrm{h}$ after inoculation. Accordingly, a $P$. syringae pv. glycinea strain (Psg R4) that does not cause disease on Arabidopsis was used as a vehicle for delivery of the HR-inducing signal controlled by avrRpt2 (Yu et al. 1993, 1998). A bulk inoculation method was designed (see Materials and Methods) that treated some but not all leaves of multiple plants. The goal was to avoid induction of a systemic HR that could eventually kill plants that exhibit an intermediate or delayed HR. Ethylmethane sulfonate (EMS) was chosen as the mutagen to enhance the likelihood of generating partial-loss-of-function alleles.

Out of approximately $11,800 \mathrm{M}_{2}$ plants screened, 209 candidate lines initially displayed a delayed or no HR. Delayed HR was defined as failure to develop the HR within $24 \mathrm{~h}$ but presence of the HR within $40 \mathrm{~h}$. Candidate plants were transplanted to larger pots, and re-inoculated with Psg R4 avrRpt2 ${ }^{+}$ 3 weeks after transplantation. Forty-two lines were then saved after giving a delayed HR or no response to Psg R4 avrRpt $2^{+}$ in the re-test. When self-progeny of these were planted for further study, 23 lines displayed a typical HR $24 \mathrm{~h}$ after inoculation and were not studied further. The $\mathrm{M}_{3}$ progeny from 12 other mutants exhibited little or no HR $40 \mathrm{~h}$ after inoculation. Seed was saved from individual $\mathrm{HR}^{-} \mathrm{M}_{3}$ plants from these lines. In order to eliminate the possibility of contamination by the mutant rps2-201 (which was planted in the original screen as a control), a pair of rps2-201-specific polymerase chain reaction (PCR) primers was used to detect the rps2-201 allele in genomic DNA of the $\mathrm{HR}^{-}$mutants. Six lines contained an rps2-201 allele and were excluded from subsequent work. The other six HR ${ }^{-}$mutants, Y3, Y5, Y15, Y16, Y23, and D25, did not contain the rps2-201 allele and were saved for further analysis (Table 1).

A weak or intermediate HR, or a mix of HR and no HR, was observed in the progeny of the final seven mutants, N8, N12, N13, N16, D8, D15, and D24 (Table 1). These lines also did not contain the rps2-201 allele. Whenever the $\mathrm{HR}^{-}$phenotype was observed in some but not all progeny derived from self-fertilization of these mutants, seeds from plants that had the clearest $\mathrm{HR}^{-}$phenotype were saved and re-tested in the next generation. All of these "intermediate-HR" mutant lines retained an intermediate $\mathrm{HR}$ phenotype in the $\mathrm{M}_{4}$ generation except N8, which was apparently isolated as an RPS $2 / r p s 2$

Table 1. HR (hypersensitive response), complementation, and growth morphology phenotypes of altered-HR mutants

\begin{tabular}{|c|c|c|c|c|c|}
\hline \multirow[b]{2}{*}{ Mutant group } & \multirow[b]{2}{*}{ Name } & \multicolumn{2}{|c|}{ avrRpt2 } & \multirow[b]{2}{*}{ Complementation of $r p s 2-201 C$} & \multirow[b]{2}{*}{ Other phenotypes } \\
\hline & & Phenotype & HR data ${ }^{a}$ & & \\
\hline \multirow{2}{*}{ Controls } & Col-0 & $\mathrm{HR}^{+}$ & $4.7 \pm 0.1$ & Yes & Wild-type parent \\
\hline & Col-0 rps $2-201 C$ & No HR & $0.1 \pm 0.0$ & No & \\
\hline \multirow[t]{2}{*}{ I } & N8 & No HR & $0.0 \pm 0.0$ & No & \\
\hline & D25 & No HR & $0.0 \pm 0.0$ & No & \\
\hline \multirow[t]{5}{*}{ II } & N12 & Mixed HR & $3.0 \pm 0.5$ & Yes & \\
\hline & N13 (ihrl) & Mixed HR & $3.2 \pm 0.2$ & Yes & \\
\hline & N16 & Mixed HR & $1.5 \pm 0.3$ & Yes & \\
\hline & D8 & Mixed HR & $3.9 \pm 0.2$ & Yes & Slight leaf narrowing \\
\hline & D24 & Mixed HR & $4.2 \pm 0.2$ & Yes & \\
\hline \multirow[t]{3}{*}{ III } & $\mathrm{Y} 16(d n d 1)$ & No HR & $0.2 \pm 0.2$ & Yes & Dwarf \\
\hline & $\mathrm{Y} 3(d n d 2)$ & No HR & $0.6 \pm 0.2$ & Yes & Dwarf \\
\hline & Y15 & Weak HR & $2.2 \pm 0.3$ & Yes & Semi-dwarf \\
\hline IV & Y23 & No HR & $0.5 \pm 0.3$ & Yes & Spontaneous lesions, dwarf \\
\hline \multirow[t]{2}{*}{ V } & Y5 & No HR & $1.7 \pm 0.7$ & Yes & Dwarf, low fertility \\
\hline & D15 & No HR & $2.2 \pm 0.3$ & Yes & Delayed development, low fertility \\
\hline
\end{tabular}

${ }^{a}$ Values are mean $\pm \mathrm{SE}$ of HR severity $(0=$ no HR, $5=$ extensive HR). All values are significantly different from HR data for Col- $0(t$ test, $P<0.05)$. 
heterozygote (see below). In summary, 13 mutant lines were saved for further study (Table 1). Our isolation and characterization of Y16, a line mutated in the DNDl gene, was previously described (Yu et al. 1998).

\section{RPS2 complementation tests.}

Crosses were made between many of the mutants and an rps 2 mutant (Col-0 rps2-201/rps2-201). The Col-0 rps2-201 line was used as a pollen donor and true crosses were verified by PCR to test genomic DNA from $\mathrm{F}_{1}$ leaf samples for presence of the rps2-201 allele. The $\mathrm{F}_{1}$ individuals from each cross were challenged with Psg R4 avrRpt2 $2^{+}$, and an HR was observed in all $\mathrm{F}_{1}$ except those from crosses with mutants $\mathrm{N} 8$ and D25. The N8 and D25 lines were considered probable rps 2 mutants and were placed in "Group I" (Table 1). However, the possibility remained that $\mathrm{N} 8$ and/or D25 carry a dominant suppressor of the HR. Therefore, $F_{2}$ plants from crosses between Col-0 rps2-201 and N8 or D25 were examined for the HR in response to Psg R4 avrRpt2 ${ }^{+}$. No HR was observed in any individuals from these $F_{2}$ populations. This was not consistent with the $3: 13$ ratio of $\mathrm{HR}^{+}: \mathrm{HR}^{-}$plants that might have been expected if N8 or D25 carried a dominant suppressor. Mutants N8 and D25 failed to complement the rps2-201 mutant; therefore, we concluded that mutants $\mathrm{N} 8$ and D25 carry rps 2 mutations.

\section{Group II: Intermediate-HR mutants.}

We identified five mutants that displayed an intermediateHR phenotype (Table 1, Group II). As an example of this phenotype, inoculation of three leaves on a given plant often produced one or two leaves showing extensive HR tissue collapse while the other one or two leaves failed to show any HR in response to the avirulent pathogen. HRs of intermediate severity were also present. This type of leaf-to-leaf variation was also observed when vacuum infiltration was used to inoculate plants. In contrast, over $95 \%$ of all leaves in control compatible and incompatible interactions gave no HR or full HR, respectively. Significantly, the mutants in Group II did not exhibit any obvious dwarfing or lesion-mimic phenotypes. Mutants N13 and D8 received the greatest attention in subsequent studies.

\section{Specificity for different avirulence genes.}

To address whether the Group II mutants N13 and D8 were altered only in the avrRpt2-RPS2 pathway, we inoculated plants with Psg R4 strains carrying other avr genes. The mutants in this study were derived from Col- 0 plants that carry a functional $R P M 1$ gene, and the avrRpml and $a v r B$ genes elicit a strong defense response in plants expressing $R P M 1$. When mutant N13 was tested, an intermediate-HR was observed in response to both Psg R4 avrRpml ${ }^{+}$and Psg R4 avrB ${ }^{+}$(Table 2). Similar results were obtained with another $P$. syringae strain, Psg R4 RUU1, and either avrRpt2, avrRpm1, or $a v r B$ (data not shown). These data suggest that the mutation in the N13 line affects a shared component of different $a v r-R$ gene pathways.

Mutant D8 also was tested with $P$. syringae carrying different avirulence genes. The HR scores observed in D8 in response to Psg R4 that express either avrRpml or avrB were not significantly different from the HR of Col-0 in response to these pathogen strains (Table 2).

\section{Growth of $P$. syringae in mutants N13 and D8.}

The HR is closely associated with gene-for-gene resistance, and loss of HR generally indicates loss of resistance to pathogens that express the $a v r$ gene. To test whether the partially compromised HR in mutants N13 and D8 was associated with defective limitation of bacterial growth, the growth of $P$. syringae pv. tomato (Pst) was monitored in both mutants. Plants were inoculated with virulent Pst DC3000 or with Pst DC3000 that express the cloned avirulence genes $a v r R p t 2^{+}, a v r B^{+}$, or avrRps $4^{+}$. Low initial doses of bacteria $\left(5 \times 10^{4} \mathrm{CFU}\right.$ per $\left.\mathrm{ml}\right)$ were applied. Mutant N13 and wild-type Col-0 displayed a similar degree of resistance to $P$. syringae that express either $a v r R p t 2$ or $a v r B$ (Fig. 1). For avirulence gene avrRps 4 and resistance gene RPS4, the resistance response of ecotype Col0 is quantitatively less effective than the response of other ecotypes such as Po-1, and is less strong than the response of Col-0 to $P$. syringae that express avirulence genes such as avrRpt 2 or avrB. (Hinsch and Staskawicz 1996; Fig. 1). When N13 plants were inoculated with Pst DC3000 that express avrRps4, we discovered an increase rather than decrease in resistance. A 10-fold reduction in Pst DC3000 avrRps $4^{+}$ population size was observed in N13 relative to Col-0 (Fig. 1).

The above result with avrRps 4 may be related to the separate finding that non-race-specific resistance to virulent $P$. syringae was also enhanced in the N13 line. Four days after inoculation, populations of virulent Pst DC3000 (no cloned $a v r$ gene) were roughly 10-fold lower in N13 relative to wild-type Col-0 (e.g., Fig. 1; reductions were twofold to 40 -fold in three independent experiments). In contrast to many previously described Arabidopsis cpr, cim, lsd, acd, and dnd mutants (Bowling et al. 1994; Dietrich et al. 1994; Dong 1998; Greenberg et al. 1994; Richberg et al. 1998; Yu et al. 1998), this elevated resistance phenotype was present in N13 despite the absence of any discernible reduction in plant size, fecundity, or leaf health.

Mutant D8 also exhibited strong resistance to Pst DC3000 that express $a v r R p t 2, a v r B$, or avrRps4 (Fig. 1). There was no significant difference between mutant D8 and Col-0 in the degree of resistance to Pst DC3000 avrRpt2 ${ }^{+}$or $a v r B^{+}$(Fig. 1). As for N13, elevated resistance to Pst DC3000 avrRps $4^{+}$was observed in mutant D8 (Fig. 1). Slightly less resistance to virulent Pst DC3000 was detected in mutant D8 than in mutant N13, but resistance to Pst DC3000 was still stronger than the wild-type Col-0 (e.g., Fig. 1; ninefold, twofold, and ninefold differences between D8 and Col-0 were observed in three independent experiments). Therefore, the weaker resistance in D8 relative to

Table 2. Hypersensitive response (HR) of selected mutants to Pseudomonas syringae expressing different avirulence genes ${ }^{\mathrm{a}}$

\begin{tabular}{llll}
\hline & \multicolumn{3}{c}{ Avirulence gene } \\
\cline { 2 - 4 } Mutant & avrRpt2 & avrRpm1 & \multicolumn{1}{c}{ avrB } \\
\hline Col-0 & $4.7 \pm 0.1$ & $4.0 \pm 0.5$ & $4.5 \pm 0.3$ \\
N13 (ihrl) & $3.2 \pm 0.2^{\mathrm{b}}$ & $2.4 \pm 0.5^{\mathrm{c}}$ & $2.6 \pm 0.6^{\mathrm{b}}$ \\
D8 & $3.9 \pm 0.2^{\mathrm{b}}$ & $3.6 \pm 0.3$ & $3.4 \pm 0.1$ \\
Y15 & $2.2 \pm 0.3^{\mathrm{b}}$ & $3.3 \pm 0.3$ & Not tested \\
Y3 (dnd2) & $0.6 \pm 0.2^{\mathrm{b}}$ & $0.0 \pm 0.0^{\mathrm{b}}$ & $0.4 \pm 0.2^{\mathrm{b}}$ \\
Y23 & $0.5 \pm 0.3^{\mathrm{b}}$ & $1.4 \pm 0.9^{\mathrm{b}}$ & $2.6 \pm 1.1^{\mathrm{b}}$ \\
\hline
\end{tabular}

${ }^{a}$ Values are mean \pm SE of HR severity $(0=$ no HR, $5=$ extensive HR) Data for avrRpt 2 are the same as those from Table 1.

${ }^{\mathrm{b}}$ Significantly different from Col-0 inoculated with same pathogen strain ( $t$ test, $P=0.05)$.

${ }^{\mathrm{c}}$ For $t$ test comparison to Col- $0, P=0.088$. 
N13 correlated with the weaker loss of HR in D8 relative to N13. As for N13, D8 exhibited this enhanced resistance with no discernible defects in plant size, fecundity, or leaf health.

\section{Disease assay.}

To further characterize the resistance response in N13 and D8, we inoculated these lines with either Pst DC3000 avrRpt $2^{+}$or Pst DC3000 and monitored macroscopic disease symptom development. Col-0, N13, and D8 were immersed into $0.01 \%$ Silwet L-77 surfactant carrying Pst DC3000 avrRpt $2^{+}$or Pst DC3000, and disease symptoms were scored 4 days after inoculation. The degree of disease was rated on a 0 to 5 scale $(0=$ no lesions, $5=$ confluent necrosis and chlorosis). Disease assay experiments were repeated on three separate dates with consistent results. Col-0, N13, and D8 all showed resistance to Pst DC3000 avrRpt2 ${ }^{+}$(average score < 0.5). Severe disease lesions were observed in Col-0 inoculated with Pst DC3000 (average score = 5). On the other hand, N13 and D8 exhibited some resistance to Pst DC3000 (average score $\approx 2$ ). This result was consistent with the elevated resistance to Pst DC3000 observed in the bacterial growth experiments (Fig. 1).

\section{Genetic analysis of N13 defines the IHR1 locus.}

Mutant N13 displayed an intermediate HR, or occasionally no HR, in response to Psg R4 avrRpt2 ${ }^{+}$. To assess dominance and to remove other unrelated mutations, N13 was backcrossed to wild-type Col- 0 . $\mathrm{F}_{1}$ plants from $\mathrm{N} 13 \times$ Col- 0
N13 (ihr1)
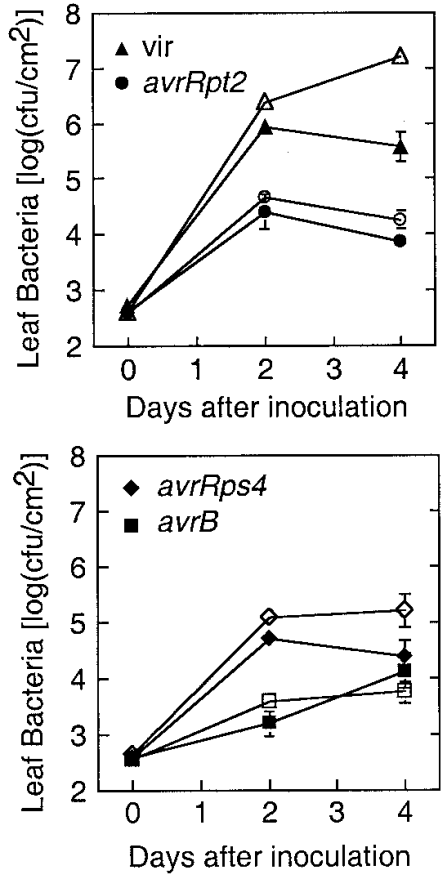

D8
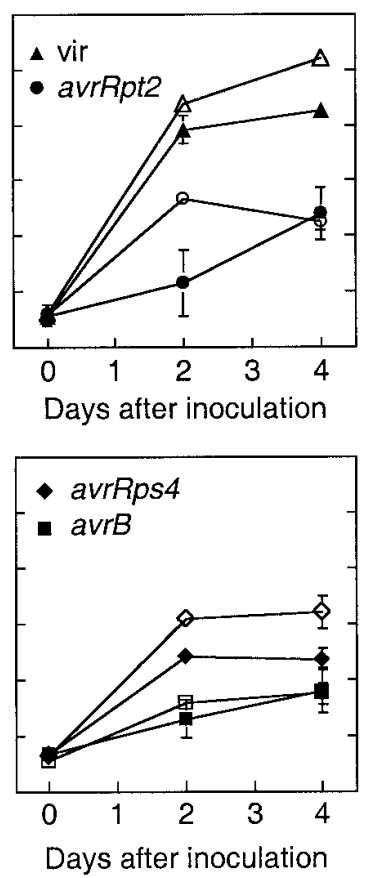

Fig. 1. Growth, in Arabidopsis Col-0 and mutants N13 (ihrl) or D8 plants, of Pseudomonas syringae pv. tomato DC3000 that express different $a v r$ genes. Six-week-old plants were inoculated with Pst DC3000 carrying pVSP61 (empty plasmid vector, "vir") or pVPS61 carrying the designated avirulence gene. Filled symbols: N13 (left panels) or D8 (right panels); open symbols: Col-0. Col-0 data from left panels are repeated in right panels to facilitate comparison. crosses exhibited a consistent HR within $24 \mathrm{~h}$ in response to Psg R4 avrRpt $2^{+}$, suggesting that $\mathrm{N} 13$ carries a recessive mutation. In an $\mathrm{F}_{2}$ population of $\mathrm{N} 13 \times \mathrm{Col}-0$, the segregation ratio for $\mathrm{HR}^{+}$: intermediate-HR: $\mathrm{HR}^{-}$was 22:10:9. However, $\mathrm{F}_{2}$ scoring was likely to be less reliable given the variable nature of the intermediate-HR phenotype and the limited number of leaves that could be assayed on each single plant. This suspicion was confirmed in progeny testing of a limited number of $\mathrm{F}_{3}$ families. For example, the $\mathrm{F}_{3}$ progeny from two independent $F_{2}$ plants that had been scored as $\mathrm{HR}^{-}$showed 19:25:3 and 18:25:6 ratios for $\mathrm{HR}^{+}$: intermediate-HR: $\mathrm{HR}^{-}$. Therefore, all subsequent conclusions regarding $\mathrm{HR}$ segregation data were based on $\mathrm{F}_{3}$ progeny test data.

For further genetic analysis of the N13 mutant, crosses were made between N13 and wild-type plants of the No-0 ecotype. A strong HR was observed in the $F_{1}$ plants. In the $F_{2}$, segregation of the HR phenotype closely resembled a $3: 1$ ratio $\left(\mathrm{HR}^{+}\right.$: intermediate-HR or $\left.\mathrm{HR}^{-}=126: 48 ; \chi^{2}=0.62, P=0.43\right) . \mathrm{F}_{3}$ segregation patterns were also consistent with a single-gene model of inheritance. In $\mathrm{F}_{3}$ progeny tests, vacuum infiltration was used to apply Psg R4 avrRpt $2^{+}$to 12 to 18 plants per $\mathrm{F}_{3}$ family. These $F_{3}$ families were subsequently scored on a 0 to 3 scale, with $0=$ No HR (similar to Col-0 rps2-201C controls), 1 = infrequent HR (similar to N13 controls), 2 = majority of plants with $\mathrm{HR}$ on most leaves, and $3=\mathrm{HR}^{+}$leaves on all plants (similar to wild-type Col-0 controls). Of $100 \mathrm{~F}_{3}$ families that were tested once, 70 families had a score of 2 or 3 and 30 families had a score of 1 . This 70:30 ratio was consistent with a $3: 1$ ratio $\left(\chi^{2}=1.33, P=0.25\right)$. An additional $57 \mathrm{~F}_{3}$ families were tested twice. Of those families, 46 exhibited a prevalent HR in at least one test while the other 11 gave infrequent HR in both tests (scores of 1 and $\leq 1$ ). This $46: 11$ ratio was consistent with $3: 1$ segregation $\left(\chi^{2}=0.98, P=0.32\right)$. The data were still consistent with a 3:1 ratio even if families that were scored once as a " 1 " and once as a " 2 " in the two tests were shifted into the reduced-HR category $\left(\chi^{2}=3.09, P=\right.$ $0.08)$. The above data therefore indicate that the N13 line carries a recessive (and incompletely penetrant) mutation at a single Mendelian locus that causes a partial or intermediate loss of HR in response to $P$. syringae that express avrRpt2. We chose the gene symbol $i h r l$ for the mutant locus in N13, indicating the phenotype of "intermediate hypersensitive response."

\section{Genetic analysis of additional Group II mutants.}

Although our original screen succeeded in identifying lines with a reproducible, reduced-HR phenotype, the intermediate or intermittent nature of the HR defect in the other Group II mutants presented a significant challenge for genetic analysis. Mutants D8, N12, and N16 were crossed to wild-type Col-0 and/or to ecotype No-0. Studies with the resulting $\mathrm{F}_{1}, \mathrm{~F}_{2}$, and $\mathrm{F}_{3}$ progeny gave results that were not inconsistent with the segregation of single recessive loci controlling the reducedHR phenotype (data not shown). However, the level of uncertainty in the scoring of individual $\mathrm{F}_{2}$ or $\mathrm{F}_{3}$ lines as $\mathrm{HR}^{+}$or reduced-HR indicated that genetic mapping would be unreliable without extensive replications, and no further genetic analysis of these lines was attempted.

\section{Group III: HR ${ }^{-/ d}$ warf mutants.}

The Group III mutants Y3 and Y16 shared many phenotypes, including a dwarfed rosette morphology and near- 
complete loss of the HR in response to $P$. syringae expressing avrRpt2, avrRpm1, or avrB. (Tables 1 and 2, Fig. 2; see also $\mathrm{Yu}$ et al. 1998). Despite the $\mathrm{HR}^{-}$phenotype of $\mathrm{Y} 3$ and $\mathrm{Y} 16$, they continued to exhibit gene-for-gene resistance (Yu et al. 1998; I.-c. Yu, R. K. Smith, K. A. Fengler, and A. F. Bent, manuscript in preparation). Accordingly, these lines were termed "defense, no death" (dnd) mutants and lines Y16 and Y3 were re-named $\bar{d} n d l$ and $d n d 2$, respectively. The $d n d 1$ mutant received the bulk of our earlier attention, and was previously described (Yu et al. 1998). Additional phenotypic and genetic analyses of the $d n d l$ and $d n d 2$ lines will be reported separately (I.-c. Yu, R. K. Smith, K. A. Fengler, and A. F. Bent, manuscript in preparation).

Y15 exhibited a less severe phenotype, with a semi-dwarfed rosette that was larger than that of Y16 (dndl) or Y3 (dnd2), and with an $\mathrm{HR}^{-}$phenotype that was more consistent than most of the Group II intermediate-HR mutants but less consistent than in $d n d 1$ or $d n d 2$. Y15 could have been placed in Group II but was placed in Group III because of the mild dwarfing of leaves/rosettes. No lesion mimic phenotype was observed in noninoculated Y15 leaves following gross inspection or microscopy of trypan blue-stained samples (data not shown).

Differences in the leaf morphology of Group III mutants extended beyond the dwarf size. Leaves of $d n d 1$, $d n d 2$, and Y15 often became slightly rolled or curled with age, and $d n d 2$ in particular often developed chlorosis at the ends (tips) of leaves during later stages of growth. Plants were otherwise quite healthy in appearance and did not exhibit other obvious developmental defects.

\section{Characterization of Y15.}

When the growth of $P$. syringae pv. tomato was monitored in Y15, these plants resembled other Group II and Group III mutants in two respects. First, despite the reduced HR, the Y15 plants continued to exhibit strong restriction of the growth of avirulent pathogens expressing avrRpt2 or avrRpm1 (Fig. 3). Second, Y15 plants exhibited enhanced resistance to virulent Pst DC3000 (Fig. 3; note that in a repeat of the experiment of Figure 3, the population size of virulent DC3000 4 days after inoculation was only 40-fold lower in Y15 relative to Col-0). Elevated resistance to virulent Pst DC3000 was also observed in surfactant-dip disease assays. In contrast to

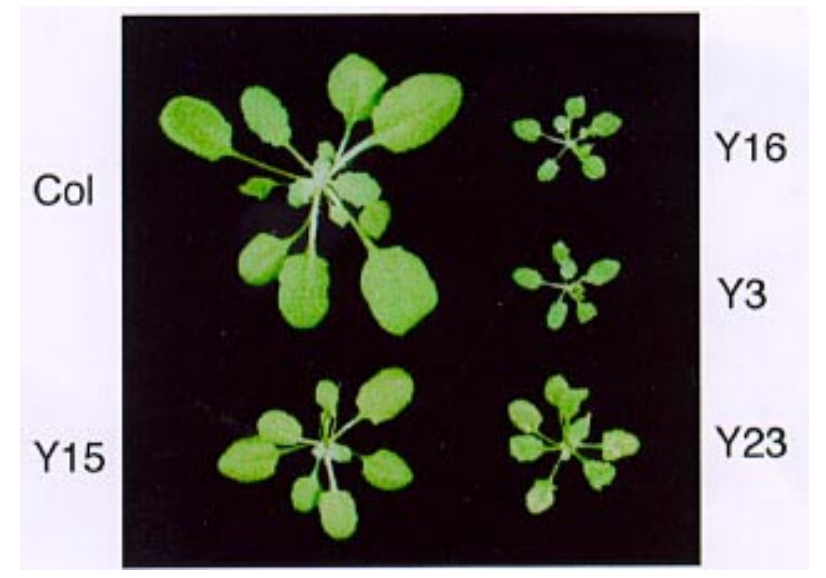

Fig. 2. Morphology of dwarfed mutant lines. Typical 5-week-old plants are shown to indicate relative sizes and appearance. the $i h r 1$ and D8 lines, for which mild disease symptoms were observed (presented above), virtually no disease symptoms were observed on Y15 plants in two separate surfactant dip experiments with virulent Pst DC3000. As might be expected, Y15 plants were highly resistant to Pst DC3000 avrRpt2 ${ }^{+}$in the surfactant dip assay. When the HR to $P$. syringae expressing different $a v r$ genes was monitored by the syringe inoculation procedure, Y15 resembled the intermediate-HR mutants rather than the $d n d$ mutants. Significant reduction of HR severity was observed in response to $P$. syringae avrRpt2 $2^{+}$, but the reduction in HR score in response to $P$. syringae that express avrRpml was not statistically significant (Table 2).

Northern (RNA) blot transcript analysis was performed with mutant Y15 to relate the intermediate-HR and elevatedresistance phenotypes to alterations in defense-related gene expression. We found that Y15 leaves exhibited elevated PR-1 transcript levels, relative to wild-type Col-0, in the absence of any inoculation with pathogen (Fig. 4; similar data obtained in repeat experiments). We also observed that pathogeninducible PR-1 gene expression was potentiated in Y15. Inoculation with virulent or avirulent $P$. syringae caused an increase in PR-1 gene expression to levels that were significantly above those observed in Col-0 (Fig. 4). In contrast, $\beta$ glucanase expression was largely unaffected in Y15; RNA levels were within twofold of wild type in all treatment conditions in multiple experiments (data not shown).

In light of the elevated disease resistance and PR-1 gene expression in Y15, we investigated the level of SA in Y15 plants. There was a clear increase in SA relative to wild-type Col-0 (Fig. 5A). Interestingly, the concentrations of SA and SA-glucoside in Y15 were roughly one-half the levels observed in $d n d 1$ and $d n d 2$ plants from the same experiments (data not shown), which correlates with the less penetrant $\mathrm{HR}^{-}$ and disease resistance phenotypes of Y15 relative to $d n d 1$ and $d n d 2$. When a 35S-nahG (salicylate hydroxylase) construct was introduced into Y15 to remove SA, the elevated disease resistance of Y15 plants was eliminated and plants were instead more susceptible than wild type (Fig. 5B).

In genetic analysis of $\mathrm{Y} 15, \mathrm{~F}_{1}$ plants of Col- $0 \times \mathrm{Y} 15$ gave a clear $\mathrm{HR}^{+}$phenotype, suggesting recessive behavior of the mutation in Y15. As with the other intermediate-HR mutants, analysis of $\mathrm{Col} \times \mathrm{Y} 15$, No- $0 \times \mathrm{Y} 15$, and other $\mathrm{F}_{2}$ populations and $\mathrm{F}_{3}$ progeny families revealed segregation of apparent true-
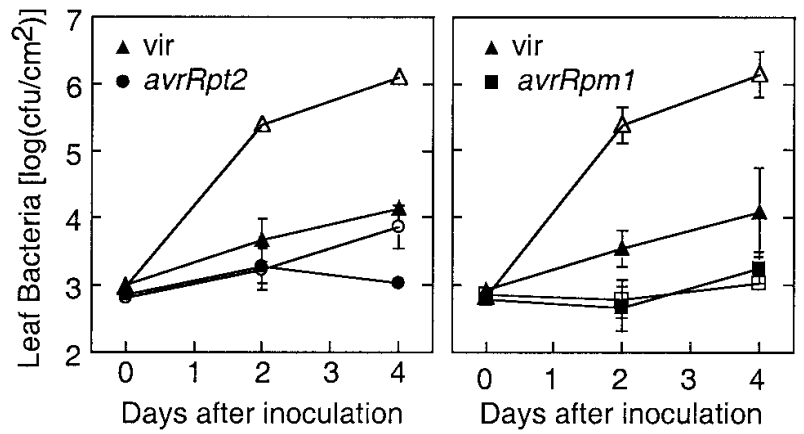

Fig. 3. Growth in Arabidopsis Col-0 and mutant Y15 plants of Pseudomonas syringae pv. tomato DC3000 carrying different avr genes. Sixweek old plants were inoculated with Pst DC3000 carrying pVSP61 (plasmid vector only) or pVPS61 carrying the designated avirulence gene. Filled symbols: Y15; open symbols: Col-0. 
breeding $\mathrm{HR}^{+}$and true-breeding reduced-HR lines. However, the reliability of the HR scoring inhibited genetic analysis at the level of precision required for genetic mapping of the mutant locus. For No- $0 \times \mathrm{Y}_{15} \mathrm{~F}_{3}$ families we also attempted to score segregation based on the partial-dwarf morphology, and based on the enhanced resistance to virulent Pst DC3000 or $P$. syringae pv. maculicola 4326. However, these phenotypes were also sufficiently intermediate or quantitative to inhibit straightforward scoring, and we concluded that extensively replicated testing would be required for reliable assignment of phenotypes to most $\mathrm{F}_{3}$ families.

\section{Resistance to virulent Peronospora parasitica.}

To examine the possibility that the intermediate-HR mutants may have an altered response to virulent pathogens other than $P$. syringae, the oomycete pathogen Peronospora parasitica was used to test the resistance of the ihrl and Y15 mutant lines. These mutants, as well as Col- 0 and Ler- 0 controls, were inoculated with $P$. parasitica Noco 2 by spraying of 9day-old seedlings. As expected (Aarts et al. 1998), Ler-0 exhibited resistance and Col- 0 exhibited susceptibility to this $P$. parasitica strain. The ihrl plants resembled Col-0 in being susceptible to $P$. parasitica, with only slightly lower fungal proliferation observed. In contrast, Y15 plants exhibited partial but clearly discernible enhanced resistance in three independent experiments. The Noco2 strain did proliferate and form haustoria on multiple cells in Y15 leaves, but this occurred at a reduced rate relative to Col- 0 controls. Sporulation levels on Y15 were 41 and $66 \%$ of wild-type Col-0 controls in the two experiments in which spore counts were taken.

\section{Group IV: HR-/dwarf/lesion-mimic mutant.}

Mutant line Y23 resembled the $d$ nd mutants in exhibiting a dwarfed rosette morphology and a failure to develop extensive hypersensitive cell death in response to $P$. syringae that express avrRpt2 (Table 1, Fig. 2). However, mutant Y23 and its progeny developed spontaneous disease lesion-like necrotic

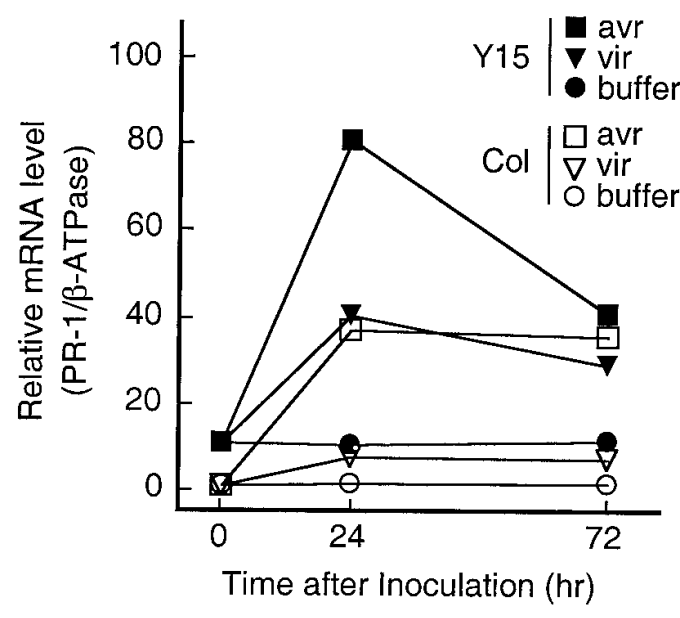

Fig. 4. PR-1 RNA levels in wild-type Col-0 and Col-0 mutant Y15 leaf tissue. Plants were inoculated with Pseudomonas syringae pv. tomato DC3000 carrying pVSP61 (plasmid vector only) or pV288 (avrRpt $2^{+}$). Hybridization of PR-1 probe was quantified with a phosphorimaging system; all data points were normalized to the level of constitutive $\beta$ ATPase transcript in the same sample, and then further normalized such that expression levels for noninoculated Col-0 $=1$. leaf flecks in the absence of inoculation with pathogen. This is similar to some $l s d$ ("lesions simulating disease") and acd ("accelerated cell death") Arabidopsis lines previously described (Dietrich et al. 1994; Greenberg et al. 1994; Richberg et al. 1998). Randomly located, isolated lesions had typically formed on lower leaves of Y23 plants by the time these plants were 4 weeks old. In contrast to the "propagation class" lesion-mimic mutants that lack the ability to stop lesion spread and ultimately display death of entire leaves and plants (Dangl et al. 1996), Y23 instead resembled the "initiation class" mutants and formed lesions of a limited size that did not engulf entire leaves. Moreover, the lesions were not enhanced by intensive light or by inoculation with avirulent $P$. syringae.

When bacterial growth was monitored within Y23 leaves, the plants exhibited enhanced resistance to both virulent and avirulent $P$. syringae (Fig. 6 and data not shown). PR-1 and $\beta$ glucanase transcript levels were constitutively elevated in Y23 (data not shown). This is similar to the constitutive resistance phenotype reported not only for other Arabidopsis lesionmimic mutants, but also for Arabidopsis cpr, cim, dnd, and ihr mutants (Bowling et al. 1994; Dietrich et al. 1994; Dong 1998; Greenberg et al. 1994; Richberg et al. 1998; Yu et al. 1998; the present work). However, of the published lesionmimic mutants, only Y23 and acd6 have been reported to exhibit an $\mathrm{HR}^{-}$phenotype (Rate et al. 1999).
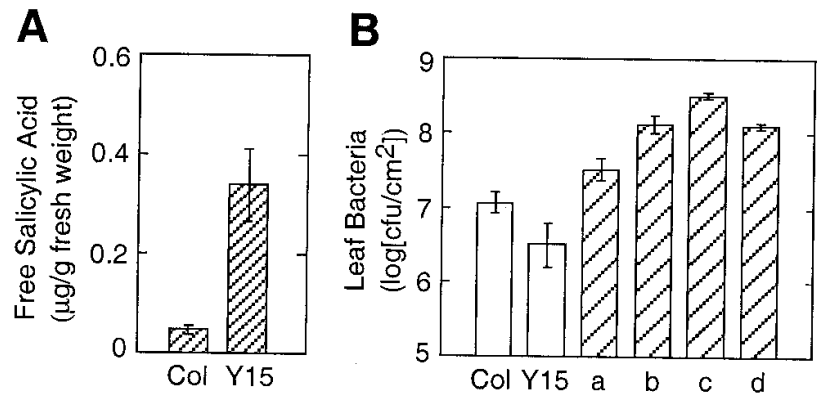

Fig. 5. A, Elevated salicylic acid (SA) levels in mutant $\mathrm{Y} 15$ as opposed to wild-type Col-0. B, Elimination of enhanced resistance to growth of Pseudomonas syringae upon expression of salicylate hydroxylase. Free SA levels were determined with noninoculated leaf tissues. Population densities of $P$. syringae pv. tomato DC3000 in leaves were determined 3 days after inoculation. Samples a, b, c, and d are four independently derived Y15 transgenic lines expressing a 35S-nahG construct.

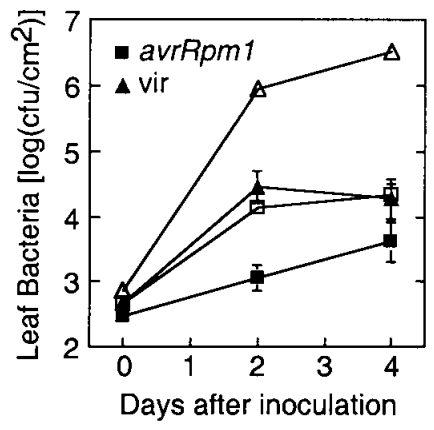

Fig. 6. Growth in Arabidopsis Col-0 and mutant Y23 plants of Pseudomonas syringae pv. tomato (Pst) DC3000 or DC3000 expressing avrRpm1. Six-week-old plants were inoculated with Pst DC3000 carrying pVSP61 (plasmid vector only) or pavrRpm1 (avrRpm $\left.1^{+}\right)$. Filled symbols: Y23; open symbols: Col-0. 
$\mathrm{F}_{1}$ plants from a $\mathrm{Y} 23 \times \mathrm{Col}-0$ cross were normal in appearance and were $\mathrm{HR}^{+}$in response to $P$. syringae expressing avrRpt2. In a $\mathrm{Y} 23 \times \mathrm{Col}-0 \mathrm{~F}_{2}$ population, the spontaneous lesion phenotype segregated in a 3:1 ratio (no lesion: lesion $=$ $\left.79: 23 ; \chi^{2}=0.33 ; P=0.57\right)$. These data indicate that the lesion-mimic trait of Y23 is controlled by a single recessive gene. However, the segregation ratio for plant size in the above $\mathrm{Y} 23 \times$ Col- $0 \mathrm{~F}_{2}$ population was not 3:1 (wild-type: dwarf morphology $=93: 9 ; \chi^{2}$ for $\left.3: 1=14.2 ; P<0.001\right)$. The presence of spontaneous lesions on $\mathrm{F}_{2}$ plants of wild-type size suggests that there is incomplete penetrance of the dwarfing phenotype in lines homozygous for the $l s d / a c d$-type mutation from line Y23.

The HR-suppression and elevated-resistance phenotypes of Y23 were temporally correlated with the onset of lesion formation. Unlike the 6- to 8-week-old Y23 plants discussed above and presented in Tables 1 and 2, the HR was readily detectable on younger Y23 plants (less than 4 weeks old) that were inoculated with avirulent $P$. syringae before spontaneous lesions had developed. When young Y23 plants with few or no spontaneous lesions were inoculated with the virulent $P$. parasitica strain Noco2, the plants resembled Col-0 in being highly susceptible to infection. This is in contrast to the $d n d$ mutants, which exhibited resistance to virulent $P$. parasitica Noco 2 and loss of the HR induced by avirulent $P$. syringae even in 3- to 4-week-old plants (Yu et al. 1998; I.-c. Yu, R. K. Smith, K. A. Fengler, and A. F. Bent, manuscript in preparation). Even in 6- to 8-week-old Y23 plants (after lesion formation), the reduction of HR in response to $P$. syringae expressing avrRpml or $a v r B$ was more quantitative or partial than for the $d n d$ mutants (Table 2).

\section{Group V: Low fertility $\mathrm{HR}^{-}$mutants.}

The Group V mutants, Y5 and D15, exhibited a reasonably clear $\mathrm{HR}^{-}$phenotype in progeny tests (Table 1). Genetic studies with these lines were initiated by backcrossing to Col-0 and outcrossing to No-0. However, the Y5 and D15 lines exhibited severely compromised fertility and received little further study.

\section{DISCUSSION}

To identify unknown components of the avrRpt2-RPS2 disease resistance pathway of Arabidopsis, we initiated a screen for mutants exhibiting a partial or complete loss of HR in response to $P$. syringae that express the avrRpt 2 gene. The screen was designed to overcome the inherent limitations of many mutational studies by allowing identification of genes that make quantitative contributions to resistance and/or genes that are lethal when fully inactive. Mutant screens often identify unexpected variants of the desired phenotype. The present screen not only produced $r p s 2$ mutants defective in resistance to $P$. syringae that express avrRpt2, but also detected mutants that are defective in the HR but competent or even enhanced for disease resistance.

Thirteen mutant lines were isolated and then divided into five groups. The first group contained two new rps 2 mutants. Group II plants, including N13 (ihrl), displayed an intermediate-HR phenotype in which $\mathrm{HR}^{+}$, intermediate $\mathrm{HR}$, and $\mathrm{HR}^{-}$ reactions could be observed on different leaves of the same plant. Group III contained defense-no-death $(d n d)$ mutants that exhibited dwarfed growth and a more complete loss of $\mathrm{HR}$, yet carried out successful gene-for-gene disease resistance. The sole mutant in Group IV was a dwarf, $\mathrm{HR}^{-}$mutant that developed spontaneous lesions prior to inoculation with pathogen. Interestingly, plants from Groups II, III, and IV also exhibited partial elevation of resistance to virulent $P$. syringae. Group V was composed of $\mathrm{HR}^{-}$plants that received little study because they produced very few seeds. The low fertility of the Group V mutants may be caused by irrelevant secondary mutations. Alternatively, these lines may demonstrate an important mutant class: highly relevant defense-related genes that are difficult to identify and study by mutation due to associated deleterious phenotypes.

The "partial function" Y15, ihrl, and other Group II mutants that we isolated exhibited $\mathrm{HR}^{+}$, intermediate-HR, and $\mathrm{HR}^{-}$phenotypes on different leaves of the same plant. The mixed or intermittent HR behavior of these lines suggests that the relevant mutations have shifted pathogen response pathways closer to some threshold for HR cell death activation. All inoculated leaves on a given plant received a similar dose of pathogen, but the mutations apparently compromised HR pathways in a way that caused subtle differences between leaves to result in very dramatic differences in HR phenotypes. Gene-for-gene disease resistance remained effective despite this altered HR phenotype (Figs. 1 and 3). We have observed a similar intermediate- or mixed-HR phenotype in transgenic Arabidopsis lines carrying RPS2 alleles with conservative amino acid substitutions in the nucleotide binding site but, unlike the present mutants, those rps 2 mutant lines exhibit less effective restriction of pathogen growth (E. Baima, D. Banerjee, and A. Bent, unpublished data). Arabidopsis phx/lsd5 double mutants that suppress cell death have been characterized by Morel and Dangl (1999) but those lines also exhibit compromised restriction of avirulent $P$. syringae.

It is worth noting that the partial-loss-of-function phenotypes of some of the mutants reported in this paper may be due to subtle or severe mutations. For example, the ihrl mutant may carry a total-loss-of-function allele that exerts a subtle effect on resistance, or IHRI may play a central role in transducing resistance signals from different $a v r / R$ gene pathways and the ihrl mutant may carry a subtle mutation in this gene.

Because ihrl exhibits similar alteration in HR and similar retention of resistance in response to different avirulent pathogens, it is likely that the ihrl mutation exerts an effect on signal transduction processes downstream of the events mediated by specific $a v r$ and $R$ (resistance) gene products. In contrast, mutants D8 and Y15 displayed an altered avrRpt2-RPS2 HR, but the intermediate HR response was statistically indistinguishable from wild-type for the avrRpm1-RPM1 pathway. However, this difference may or may not indicate that the mutations in D8 and Y15 alter a gene with functional specificity for the avrRpt2-RPS2 pathway. RPM1 mediates an earlier/stronger HR than RPS2 in wild-type plants (Ritter and Dangl 1996; I.-c. Yu and A. F. Bent, unpublished data). It may be the case that loss or inhibition of the HR in the D8 and Y15 mutants is too weak to alter the $R P M 1$-mediated response.

Significantly, the ihrl, D8, and Y15 mutants exhibited a modest but highly reproducible increase in resistance to virulent P. syringae (Figs. 1 and 3; and data not shown). While ihrl, D8, and Y15 still developed some disease symptoms 
(necrotic lesions and chlorosis), the extent of disease was substantially lower than in Col-0. Y15 also exhibited elevated resistance to a second pathogen, the virulent $P$. parasitica strain Noco2. The partial elevation of resistance in Y15 correlated with elevated PR-1 expression and elevated levels of SA. The dependence of resistance on SA was shown in Y15 nah $G^{+}$lines.

In addition to the enhanced resistance to virulent $P$. syringae, elevated resistance in the avrRps4-RPS4 pathway was observed. Wild-type Col-0 plants only exhibit weak restriction of the growth of $P$. syringae that express avrRps 4 and only a weak HR in response to these strains, providing a particularly sensitive system with which to detect altered defense responses (Hinsch and Staskawicz 1996; and this work). The enhanced resistance in the $i h r l$ and D8 mutant lines both to virulent $P$. syringae and to $P$. syringae that express avrRps 4 suggests a relationship between enhanced resistance to virulent pathogens and potentiation of gene-for-gene signaling. These data provide further evidence of the overlap that has been observed between gene-for-gene and more general defense responses (Delaney et al. 1994; Richberg et al. 1998; Shirasu et al. 1997). The HR apparently reinforces or enhances defense activation in plant tissues (Alvarez et al. 1998; Dangl et al. 1996), but in intermediate-HR mutants the elevated constitutive defenses may substitute for the HR and foster an analogous enhancement of gene-for-gene defenses.

Unlike previously reported Arabidopsis lines with elevated resistance such as many of the $c p r$, cim, lsd, and acd mutants and our dnd mutants (Dong 1998; Richberg et al. 1998; Yu et al. 1998), the elevated resistance in ihrl and D8 occurred without dwarfing of the plants. This may be due to a significant difference in the mechanism of defense activation in $i h r l$ and D8 as opposed to the previous mutants. Alternatively, the absence of dwarfing may be a quantitative phenomenon that correlates with the weaker elevation of nonspecific resistance in $i h r l$ and D8 as opposed to the dwarfed mutants, and that correlates with the less complete loss of HR in the ihrl and D8 mutants. Consistent with this correlation, Y15 plants did exhibit partial dwarfing, and also exhibited heightened resistance to $P$. parasitica that was not observed in the $i h r l$ line. Despite the intentional design of our mutant screen to detect intermediate phenotype mutants, difficulties with reliable phenotypic scoring of segregating progeny made precise genetic analysis of these partial-function mutants a challenging task. However, strong agricultural biotechnology opportunities could be revealed through identification and study of genes that elevate resistance without associated penalties in plant growth and productivity.

In contrast to $i h r l$, D8, and the other Group II mutants, the dnd (Y3 and Y16) and $l s d / a c d$ (Y23) mutants that we isolated exhibited a more complete loss of HR, an obvious dwarfed phenotype, and a more strongly elevated resistance against virulent $P$. syringae. These similarities between our $d$ nd and $l s d / a c d$ mutants are interesting in light of their obviously different lesion-mimic phenotypes. It is also interesting that many previously isolated dwarf/elevated resistance mutants (including many $c p r, c i m, l s d$, and acd mutants) still generate HR cell death in response to avirulent $P$. syringae, while the HR is largely absent in the dnd and lsd/acd mutants that we isolated. These differences imply that the metabolic alterations caused by these various mutations, while partially overlapping, also differ in significant ways.
The simultaneous presence of the reduced HR and elevated general resistance phenotypes is perhaps not surprising, given previous evidence for feedback loops that link necrotic cell death and induction of SAR and local resistance (Dangl et al. 1996; Ryals et al. 1996). The ihr, dnd, and other mutants reported in this study may activate defense in a way that secondarily activates cell death suppression or otherwise interferes with the HR. However, it is also possible that the reduced-HR mutations first disrupt the HR and/or other cell death pathways, and secondarily activate defenses. The molecular mechanisms that underlie the phenotypes of the presently reported mutants remain to be discovered. A number of biochemical processes have been implicated in defense signaling, including the action of protein kinases, SA, reactive oxygen species, nitric oxide, and calcium-mediated signaling events (Delledonne et al. 1998; Durner et al. 1998; HammondKosack and Jones 1996; Scheel 1998). Perturbation of these or other processes might account for the observed alterations in defense activation and HR. The $i h r, d n d$, and related mutants isolated in the present study provide an entry point for future research, and further study of these lines should reveal new information on the structure and regulation of pathogen defense and cell death pathways in plants.

\section{MATERIALS AND METHODS}

\section{Pathogens.}

P. syringae pv. glycinea race 4 (Psg R4) and RUU1 (another race 4 strain) were originally obtained from $N$. T. Keen of the University of California-Riverside. P. syringae pv. tomato (Pst) DC3000 was originally provided by D. Cuppels of the University of Ontario. The plasmids pV288, pavrRpm1, pVB01, and pKec 218 carry the $a v r$ genes avrRpt2, avrRpm1, avrB, and avrRps4, respectively, on the plasmid pVSP61 (W. Tucker, DNA Plant Technology, Oakland, CA; Bisgrove et al. 1994; Hinsch and Staskawicz 1996; Kunkel et al. 1993). The plasmids were introduced into Psg or Pst by tri-parental mating with the helper plasmid pRK2013 (Figurski and Helinski 1979). $P$. syringae were grown at $28^{\circ} \mathrm{C}$ either in NYG broth $(5$ $\mathrm{g}$ of Bactopeptone, $3 \mathrm{~g}$ of yeast extract, and $20 \mathrm{ml}$ of glycerol per liter; Daniels et al. 1984) or on NYGA plates (NYG + $1.5 \%$ agar) with kanamycin $(25 \mu \mathrm{g} / \mathrm{ml})$ and/or Rifampicin 50 $\mu \mathrm{g} / \mathrm{ml}$ ). Cells were resuspended to the specified density in 10 $\mathrm{mM} \mathrm{MgCl}{ }_{2}$ prior to use for plant inoculation.

Spores from $P$. parasitica Noco2 were resuspended in $\mathrm{H}_{2} \mathrm{O}$ at $4 \times 10^{4}$ spores per $\mathrm{ml}$ and sprayed on 9-day-old seedlings (Parker and Coleman 1997). $\mathrm{H}_{2} \mathrm{O}$ was used as a negative control. The inoculated seedlings were grown in a growth chamber with nearly $100 \%$ humidity at $18^{\circ} \mathrm{C}$. Macroscopic phenotypes were monitored at days 3,5 , and 6 .

\section{Mutant screen.}

A screening method, previously described in brief (Yu et al. 1998), was designed to allow infiltration of $P$. syringae into some but not all leaves of large numbers of plants. Arabidopsis thaliana ecotype Columbia (Col-0) ethylmethane-sulfonate (EMS) mutagenized $\mathrm{M}_{2}$ seeds were obtained from Lehle Seeds (Round Rock, Texas). Plants were grown in 50-cmlong, 1.2-cm-diameter polyvinyl chloride (PVC) tubing that had been split longitudinally to leave approximately $60 \%$ of the tube circumference. Tubes were capped at the ends, drain 
holes were added, and tubes were filled with potting soil (Sunshine mix \#1; Sun Gro Horticulture, Bellevue, WA). Approximately $50 \mathrm{M}_{2}$ seeds, as well as 3 Col-0 and 3 Col-0 rps 2 201/rps2-201 seeds as controls, were planted in each tube. All seeds were stratified at $4^{\circ} \mathrm{C}$ for 2 days before being placed in a growth chamber. The growth chamber was set for a $9 \mathrm{~h}$ light $/ 15 \mathrm{~h}$ dark photoperiod at $22^{\circ} \mathrm{C}$. After 2 weeks, plants were transferred to continuous light for another 4 weeks. Twenty minutes before infiltration with pathogen, approximately $25 \mathrm{ml}$ of $0.5 \%$ Bacto-Agar (Difco, Detroit, MI.) was added on the top of the soil to prevent loss of soil during the infiltration process. To inoculate plants, the tubes with growing plants were rotated axially $90^{\circ}$ and set in plasticene endmounts in a trough made of 5-cm-diameter, 60-cm-long PVC tube that had been split longitudinally, capped at the end, and filled with a solution of $P$. syringae pv. glycinea race 4 (Psg R4) pV288 (avrRpt2 $\left.2^{+}\right)$at a concentration of $2 \times 10^{8}$ CFU per $\mathrm{ml}$. Approximately half of the leaves on each plant were immersed in pathogen solution. Entire set-ups were then placed in a large vacuum chamber, a vacuum was drawn for about 2 min or until the solution was bubbling vigorously, and the vacuum was then released. Inoculated plants were scored visually for development of HR-associated tissue collapse 24 and $40 \mathrm{~h}$ after inoculation. $\mathrm{M}_{2}$ plants that exhibited either a delayed HR or no HR on inoculated leaves were transplanted to standard pots and soil for further testing. These putative mutants were re-inoculated with Psg R4 and Psg R4 avrRpt2 ${ }^{+}$ 3 weeks after being transplanted (see HR assay below). The mutants that retained the altered-HR phenotype in response to Psg R4 avrRpt $2^{+}$were saved and grown to maturity for seeds. Backcrosses of mutants to wild-type Col-0 were pursued with original $\mathrm{M}_{2}$ plants. Progeny plants were grown in $9 \mathrm{~h}$ light/15 $\mathrm{h}$ dark at $22^{\circ} \mathrm{C}$ in a growth chamber for 6 weeks prior to further testing.

\section{Genetic analysis.}

Crosses were made between the putative mutants and Col0, Col-0 gl-1/gl-1, an rps2 mutant (Col-0 rps2-201/rps2-201), and Nössen (No-0) to perform dominance, complementation, and inheritance tests, and to reduce the presence of secondary mutations. To verify crosses, PCR was performed as in Ausubel et al. (1997) with Arabidopsis genomic DNA and primers RPS2-48-2 (5'-GCTCCTCCTAAAGTGAT), D203RPS2 (5'-TACTTGGAAAACCTAC), and RPS2-46Sal (5'AGACGGTCGACAAATGGATTTCATCTCA) to detect the rps2-201C allele (Bent et al. 1994), or nga106F (5'GTTATGGAGTTTCTAGGGCACG) and nga106R (5'TGCCCCATTTTGTTCTTCTC) to distinguish between ecotypes (Bell and Ecker 1994). In each experiment, separate reactions were performed with genomic DNA from reference plant genotypes as positive and negative controls. Allelism between new mutations and RPS 2 was detected in $\mathrm{F}_{1}$ plants as failure to complement the Col-0 rps2-201/rps2-201 line for $\mathrm{HR}$ in response to Psg R4 avrRpt2 $2^{+}$. Inheritance of the altered$\mathrm{HR}$ phenotype and other traits was examined in $\mathrm{F}_{1}, \mathrm{~F}_{2}$, and $\mathrm{F}_{3}$ populations from the above crosses.

\section{HR, disease, and bacterial growth assays.}

HR assays were performed as previously described (Klement et al. 1964; Yu et al. 1998) with pipette or vacuum infiltration used to inoculate plants with 1 to $2 \times 10^{8} \mathrm{CFU}$ per $\mathrm{ml}$ of Psg R4 expressing avr genes to induce visible leaf collapse (= HR) in incompatible interactions. All HR assays included Psg R4 pVSP61 (no avr gene) as a control. Data reported in Tables 1 and 2 represent cumulative values from multiple experiments. Surfactant dip disease assays used Pst strains resuspended in $0.01 \%$ Silwet L-77, $10 \mathrm{mM} \mathrm{MgCl}_{2}$ as described (Whalen et al. 1991). For disease assays with syringes or pipettes, plants were inoculated with Pst DC3000 avrRpt $2^{+}$or Pst DC3000 at $5 \times 10^{5} \mathrm{CFU}$ per $\mathrm{ml}$ and disease was scored 4 days after inoculation. Bacterial growth in leaves was monitored as described following vacuum infiltration of Pst strains resuspended at $5 \times 10^{4} \mathrm{CFU}$ per $\mathrm{ml}$ (Whalen et al. 1991).

\section{RNA blots and SA.}

RNA blots were performed with inoculated and noninoculated leaf tissue samples as described (Yu et al. 1998). SA levels were determined on triplicate samples of mature leaf tissue as described (Uknes et al. 1993). Y15 lines expressing $n a h G$ (salicylate hydroxylase) under control of a double-35S promoter were obtained by floral dip transformation (Clough and Bent 1998) with pCIB200 (Gaffney et al. 1993); data are reported for $\mathrm{T}_{3}$-generation plants from four independent transgenic lines homozygous for a single-locus transgene insertion.

\section{ACKNOWLEDGMENTS}

We thank Jane Parker for performing many of the Peronospora parasitica assays, Novartis (NABRI) for providing pCIB200 and facilities for salicylic acid studies, Thom Hoffman, J. Scott Schmidt, and Gracia Zabala for help with mutant screening and analysis, and Grace Jurkowski for critical review of the manuscript.

\section{NOTE ADDED IN PROOF}

At our new location in Wisconsin we have observed infrequent lesion mimic spotting on leaves of a small minority of Y16 (dnd1) and Y3 (dnd2) plants. Some but not all colleagues who have grown Y16 and Y3 plants at other institutions have also observed these lesions, which are apparently elicited by an unidentified environmental factor.

\section{LITERATURE CITED}

Aarts, N., Metz, M., Holub, E., Staskawicz, B. J., Daniels, M. J., and Parker, J. E. 1998. Different requirements for EDS1 and NDRl by disease resistance genes defined at least two $R$ gene-mediated signaling pathways in Arabidopsis. Proc. Natl. Acad. Sci. USA 95:1030610311.

Alvarez, M. E., Pennell, R. W., Meijer, P.-J., Ishikawa, A., Dixon, R. A., and Lamb, C. 1998. Reactive oxygen intermediates mediate a systemic signal network in the establishment of plant immunity. Cell 92: 773-784.

Ausubel, F. M., Brent, R., Kingston, R. E., Moore, D. D., Seidman, J. G., Smith, J. A., and Struhl, K. 1997. Current Protocols in Molecular Biology. John Wiley \& Sons, New York.

Bell, C. J., and Ecker, J. R. 1994. Assignment of 30 microsatellite loci to the linkage map of Arabidopsis. Genomics 19:137-144.

Bendahmane, A., Kanyuka, K., and Baulcombe, D. C. 1999. The $R x$ gene from potato controls separate virus resistance and cell death responses. Plant Cell 11:781-791.

Bent, A. F. 1996. Plant disease resistance genes: Function meets structure. Plant Cell 8:1757-1771.

Bent, A. F., Kunkel, B. N., Dahlbeck, D., Brown, K. L., Schmidt, R. L., Giraudat, J., Leung, J. L., and Staskawicz, B. J. 1994. RPS2 of Arabidopsis thaliana: A leucine-rich repeat class of plant disease resistance genes. Science 265:1856-1860.

Bisgrove, S. R., Simonich, M. T., Smith, N. M., Sattler, A., and Innes, R. 
W. 1994. A disease resistance gene in Arabidopsis with specificity for two different pathogen avirulence genes. Plant Cell 6:927-933.

Bowling, S. A., Guo, A., Cao, H., Gordon, A. S., Klessig, D. F., and Dong, X. 1994. A mutation in Arabidopsis that leads to constitutive expression of systemic acquired resistance. Plant Cell 6:1845-1857.

Clough, S. J., and Bent, A. F. 1998. Floral dip: A simplified method for Agrobacterium-mediated transformation of Arabidopsis thaliana. Plant J. 16:735-743.

Dangl, J. L., Dietrich, R. A., and Richberg, M. H. 1996. Death don't have no mercy: Cell death programs in plant-microbe interactions. Plant Cell 8:1793-1807.

Daniels, M. J., Barber, C. E., Turner, D. C., Cleary, S. G., and Sawzyc, M. K. 1984. Isolation of mutants of Xanthomonas campestris pv. campestris showing altered pathogenicity. J. Gen. Microbiol. 130: 2447-2455.

Delaney, T. P., Uknes, S., Vernooij, B., Friedrich, L., Weymann, K., Negrotto, D., Gaffney, T., Gutrella, M., Kessmann, H., Ward, E., and Ryals, J. 1994. A central role of salicylic acid in plant disease resistance. Science 266:1247-1250.

Delledonne, M., Xia, Y., Dixon, R. A., and Lamb, C. 1998. Nitric oxide functions as a signal in plant disease resistance. Nature 394:585-588.

Dietrich, R. A., Delaney, T. P., Uknes, S. J., Ward, E. R., Ryals, J. A., and Dangl, J. L. 1994. Arabidopsis mutants simulating disease resistance response. Cell 77:565-577.

Dong, X. 1998. SA, JA, ethylene and disease resistance in plants. Curr Opin. Plant Biol. 1:316-323.

Dong, X., Mindrinos, M., Davis, K. R., and Ausubel, F. M. 1991. Induction of Arabidopsis thaliana defense genes by virulent and avirulent Pseudomonas syringae strains and by a cloned avirulence gene. Plant Cell 3:61-72.

Durner, J., Wendehenne, D., and Klessig, D. F. 1998. Defense gene induction in tobacco by nitric oxide, cyclic GMP, and cyclic ADPribose. Proc. Natl. Acad. Sci. USA 95:10328-10333.

Figurski, D. H., and Helinski, D. R. 1979. Replication of an origincontaining derivative of plasmid RK2 dependent on a plasmid function provided in trans. Proc. Natl. Acad. Sci. USA 76:1648-1652.

Gaffney, T., Friedrich, L., Vernooij, B., Negrotto, D., Nye, G., Uknes, S., Ward, E., Kessman, H., and Ward, J. 1993. Requirement of salicylic acid for the induction of systemic acquired resistance. Science 261:754-756.

Goodman, R. N., and Novacky, A. J. 1994. The Hypersensitive Reaction in Plants to Pathogens: A resistance Phenomenon. American Phytopathological Society, St. Paul, MN.

Goulden, M. G., and Baulcombe, D. C. 1993. Functionally homologous host components recognize potato virus X in Gomphrena globosa and potato. Plant Cell 5:921-930.

Greenberg, J. T., Guo, A. L., Klessig, D. F., and Ausubel, F. M. 1994. Programmed cell death in plants - a pathogen-triggered response activated coordinately with multiple defense functions. Cell 77:551-563.

Hammond-Kosack, K. E., and Jones, J. D. G. 1996. Resistance genedependent plant defense responses. Plant Cell 8:1773-1791.

Hinsch, M., and Staskawicz, B. 1996. Identification of a new Arabidopsis disease resistance locus, RPS4, and cloning of the corresponding avirulence gene, avrRps4, from Pseudomonas syringae pv. pisi. Mol. Plant-Microbe Interact. 9:55-61.

Innes, R. W. 1998. Genetic dissection of R gene signal transduction pathways. Curr. Opin. Plant Biol. 1:299-304.

Klement, Z., Farkas, G. I., and Lovrekovich, L. 1964. Hypersensitive reaction induced by phytopathogenic bacteria in the tobacco leaf. Phytopathology 54:474-477.

Kunkel, B. N., Bent, A. F., Dahlbeck, D., Innes, R. W., and Staskawicz, B. J. 1993. RPS2, an Arabidopsis disease resistance locus specifying recognition of Pseudomonas syringae strains expressing the avirulence gene avrRpt2. Plant Cell 5:865-875.

Lehnackers, H., and Knogge, W. 1990. Cytological studies on the infection of barley cultivars with known resistance genotypes by Rhynchosporium secalis. Can. J. Bot. 68:1953-1961.

Morel, J. B., and Dangl, J. L. 1999. Suppressors of the Arabidopsis lsd 5 cell death mutation identify genes involved in regulating disease resistance responses. Genetics 151:305-19.

Ori, N., Eshed, Y., Paran, I., Presting, G., Aviv, D., Tanksley, S., Zamir, D., and Fluhr, R. 1997. The $I 2 C$ family from the wilt disease resistance locus I 2 belongs to the nucleotide binding, leucine-rich repeat superfamily of plant resistance genes. Plant Cell 9:521-532.

Parker, J. E., and Coleman, M. J. 1997. Molecular intimacy between proteins specifying plant-pathogen recognition. Trends Biochem. Sci. 22:291-296.

Rate, D. N., Cuenca, J. V., Bowman, G. R., Guttman, D. S., and Greenberg, J. T. 1999. The gain-of-function Arabidopsis acd6 mutant reveals novel regulation and function of the salicylic acid signaling pathway in controlling cell death, defenses, and cell growth. Plant Cell 11:1695-1708.

Richberg, M. H., Aviv, D. H., and Dangl, J. L. 1998. Dead cells do tell tales. Curr. Opin. Plant Biol. 1:480-485.

Ritter, C., and Dangl, J. L. 1996. Interference between two specific pathogen recognition events mediated by distinct plant disease resistance genes. Plant Cell 8:251-257.

Ryals, J. L., Neuenschwander, U. H., Willits, M. C., Molina, A., Steiner, H.-Y., and Hunt, M. D. 1996. Systemic acquired resistance. Plant Cell 8:1809-1819.

Scheel, D. 1998. Resistance response physiology and signal transduction. Curr. Opin. Plant Biol. 1:305-310.

Schiffer, R., Görg, R., Jarosch, B., Beckhove, U., Bahrenberg, G., Kogel, K.-H., and Schulze-Lefert, P. 1997. Tissue dependence and differential cordycepin sensitivity of race-specific resistance responses in the barley-powdery mildew interaction. Mol. Plant-Microbe Interact. 10: 830-839.

Shirasu, K., Nakajima, H., Rajasekhar, V. K., Dixon, R. A., and Lamb, C. J. 1997. Salicylic acid potentiates an agonist-dependent gain control that amplifies pathogen signals in the activation of defense mechanisms. Plant Cell 9:261-270.

Uknes, S., Winter, A. M., Delaney, T., Vernooij, B., Morse, A., Friedrich, L., Nye, G., Potter, S., Ward, E., and Ryals, J. 1993. Biological induction of systemic acquired resistance in Arabidopsis. Mol. PlantMicrobe Interact. 6:692-698.

Whalen, M., Innes, R., Bent, A., and Staskawicz, B. 1991. Identification of Pseudomonas syringae pathogens of Arabidopsis thaliana and a bacterial gene determining avirulence on both Arabidopsis and soybean. Plant Cell 3:49-59.

Yu, G.-L., Katagiri, F., and Ausubel, F. M. 1993. Arabidopsis mutations at the RPS2 locus result in loss of resistance to Pseudomonas syringae strains expressing the avirulence gene avrRpt2. Mol. Plant-Microbe Interact. 6:434-443.

Yu, I.-c., Parker, J., and Bent, A. F. 1998. Gene-for-gene disease resistance without the hypersensitive response in Arabidopsis dndl mutant. Proc. Natl. Acad. Sci. USA 95:7819-7824. 